\title{
Oncolytic Vaccinia Virus TG6002
}

National Cancer Institute

\section{Source}

National Cancer Institute. Oncolytic Vaccinia Virus T G6002. NCI Thesaurus. Code C148178.

A modified, replicative oncolytic vaccinia virus (VV), deleted of the genes for tyrosine kinase (TK) and ribonucleotide reductase (RR), and expressing the yeast-originated, bifunctional cytosine deaminase/uracil phosphoribosyltransferase gene (FCU1), with potential immunomodulating and antineoplastic activities. Upon intravenous administration of the oncolytic vaccinia virus T G6002, the virus preferentially targets and infects tumor cells, causing oncolysis. In turn, the lysed tumor cells release various tumor-associated antigens (TAAs), which induce an immune response against the tumor cells. Upon concomitant administration of the non-cytotoxic prodrug flucytosine (5fluorocytosine; 5-FC), the FCU1 expressed in the infected cancer cells produce the enzymes cytosine deaminase and uracil phosphoribosyltransferase which catalyze the conversion of 5-FC into the cytotoxic forms 5-fluorouracil (5-FU) and 5-fluoro-uridilyl monophosphate (5-FUMP); 5-FU and 5-FUMP exert a cytotoxic effect in the infected tumor cells. Double gene deletion (TK-RR-) restricts the propagation of T G6002 to the tumor cells, thereby reducing toxicity to normal cells. 